\title{
Multi-scale Modelling of Sintering
}

\author{
Jingzhe Pan* and Ruoyu Huang \\ Department of Engineering, University of Leicester \\ Leicester, LE1 7RH, UK; e-mail: jp165@le.ac.uk \\ * The corresponding author
}

\begin{abstract}
Sintering models at the atomistic, particle and component scales are briefly reviewed and problems in the current generation of sintering models are highlighted. A strategy of multi-scale modelling is proposed and our progress in the multiscale modelling is reported. Mathematical details of the models are left out and the paper is set out to demonstrate what is possible in the next generation of sintering models.
\end{abstract}

\section{Introduction}

Sintering is a process in which powder compacts are fired and consolidated into strong solids. Driven by the reduction in surface energy, a porous material shrinks and consolidates at elevated temperatures. Sintering can be achieved by a range of mechanisms including viscous flow, solid state diffusion, vapour evaporation/condensation, or formation of a small amount of liquid phase. Most ceramic products are made by sintering. Accurately predicting the shrinkage and microstructure of sintered products is very useful to ceramic manufacturers. However modelling sintering is one of the most challenging problems in material modelling. Sintering deformation is fundamentally linked to microstructural evolution and depends on very subtle changes in microstructure and chemistry, sometimes at the atomic level. Consequently, the ability of prediction by the current generation of sintering models (using the continuum finite element analysis for example) is poor. On the other hand, this challenge has provided an ideal platform for integrating modelling techniques at the atomistic, particle and continuum levels. Bringing together multi-scale elements to create an integrated sintering model is the theme of this paper. The integrated model represents a significant step forward in improving the predictive capability of the sintering models.

\section{An overview of sintering models at different length scales}

Sintering models have been developed at the atomistic, particle and component scales which were recently reviewed by Pan [1]. Here a brief overview and update is provided. A full reference list can be found in [1].

Atomistic modes: One of the major developments in materials chemistry during the past two decades is the growing ability of computational techniques to model accurately the behaviour of solid materials at the atomistic level. Molecular dynamics is one such technique which provides a detailed picture of the evolution of atomic positions and velocities as a function of time based on Newton's equations of motion. The atomic interactions are characterised by the inter-atomic potentials, hence the chemical composition of the material is explicit in the model. The thermodynamic and kinetic properties of the material can be extracted from the simulation results. For example, one can calculate the diffusion coefficient using the time-dependent mean square displacements. It is a powerful tool to understand the effect of impurities and doping on diffusion coefficients and interfacial energies, which are the fundamental parameters controlling the sintering process. Currently direct molecular dynamic simulation of particle sintering is only possible for nano-sized particles because of their extreme demand for computing power. Such simulations have been performed by several authors which were basically people's first attempts to show that this could be done[2].

Particle models: Sintering models at the particle scale have come a long way since the classical works by early pioneers such as Frenkel, Kuczynski, Herring, Coble, Kingery and et al. Two major advances have been made, mainly due to the growing ability of computational techniques. The first one is that the discrete element technique has been developed to model the interaction between thousands of particles[3]. This technique is however limited to sintering at the very early stage because it relies on contact laws which assume isolated contacts. The second one is that a variational numerical technique 
has been developed which is able to follow the detailed microstructural evolution of particle compacts consisting of thousands of particles of arbitrary shapes and sizes to a fully dense solid[4,5]. At the core of the microscopic model are the kinetic laws governing solid-state diffusion and grain-boundary migration. A material is characterised by the specific surface and grain-boundary energies, diffusion coefficients and grain-boundary mobility. The micro-mechanical model can handle agglomeration, particle/grain size/pore distributions and anisotropy, but it cannot directly model the effect of impurities and doping. Their influence on the microstructural evolution is reflected in the diffusion coefficient and interfacial energies. Currently the variational model is limited to solid state and viscous sintering.

Continuum models: The finite element method can be used to calculate sintering deformation of components of sophisticated shapes. At the core of the finite element analysis are the constitutive laws for the material response. At the macroscopic scale, the sintering process can be considered as a viscous deformation of a continuum solid driven by a self-existing stress. The fundamental equation governing the viscous deformation is the stress - strain rate relation, known as the constitutive law. For micron-sized particles, a linear constitutive law has been found to be valid for a large range of powder materials:

$$
\dot{\varepsilon}_{i j}=\frac{s_{i j}}{2 \eta_{S}}+\frac{\sigma_{m}}{3 \eta_{B}} \delta_{i j}-\frac{\sigma_{s}}{3 \eta_{B}} \delta_{i j},
$$

in which $\dot{\varepsilon}_{i j}$ is the strain rate, $s_{i j}$ and $\sigma_{m}$ the devitoric and mean stresses, $\sigma_{s}$ the self-existing stress known as the sintering potential, $\eta_{S}$ the shear viscosity, $\eta_{B}$ the bulk viscosity, and $\delta_{i j}$ the Kronecker delta function. The simplicity of eqn. (1) is deceiving because the two viscosities are strong functions of temperature and microstructure, both changes significantly in the sintering process. To use the constitutive law in a finite element analysis, the dependence of the viscosities and the sintering potential on the temperature and microstructure must be known. There are two ways of obtaining this dependence: (a) using a micromechanical (particle scale) material model and (b) directly fitting the experimental data. Various micromechanical models have been proposed. However their predictions are so diverse that it is difficult to know which one to use. Directly measuring the viscosities has been done by several research groups [6]. The measurement is however full of pitfalls. A force has to be applied to the fragile sample at high temperature to measure the viscosities and the sintering potential. This very force can alter the microstructure and lead to a viscosity for an unwanted microstructure (a highly anisotropic one for example). The viscosities and sintering potential also have to be measured across the full range of sintering temperature and density which is very time-consuming. The tensile viscosity of a powder compact is very different from the compressive one; a particle cluster has a much higher resistance to compression than to tension. Applying a tensile force to a fragile powder compact is even more difficult and we are not aware of any such measurement. Conventional constitutive models of sintering are expressed in terms of the evolution of two scalar state variables, density and grain-size. They do not provide any information about how changes in initial particle size distributions or agglomeration influence the constitutive response. Also, they do not provide information about the influence of chemical impurity and doping on the kinetic constants and how this affects the details of the densification and grain-growth processes. Most of the models assume that the response is isotropic. The anisotropic structure developed during compaction can have a strong influence on deformation and grain-growth during sintering. It is important to fully model material anisotropy and its influence on distortion of a component during sintering. Because of these difficulties, finite element calculation of sintering deformation has not been widely used in the ceramic industry.

Compaction models: The sintering process cannot be modelled in isolation. Non-uniform initial density and residual stresses induce differential sintering, distortion and even cracks. It is extremely important to provide the sintering analysis with reliable input for the initial material state and residual stresses. This requires the development of computational models of the compaction process whose output is compatible with the requirements of the sintering models. A range of constitutive models have been developed for compaction [7]. Like the sintering models described above, conventional 
models assume that the state can be described in terms of the density. It is then relatively straightforward to combine the compaction model with sintering models which employ the same state variables.

Model integration: Table one summarises the input and output of sintering models at atomistic, particle and macroscopic scales respectively.

Table one - Input and output of sintering models at different length scales.

\begin{tabular}{|c|c|c|}
\hline & Model input & Model output \\
\hline Atomistic scale & $\begin{array}{l}\text { 1. Interatomic potential \& its parameters } \\
\text { 2. Initial crystalline structure } \\
\text { 3. Temperature }\end{array}$ & $\begin{array}{l}\text { 1. Trajectory and velocity of each atom } \\
\text { 2. Specific interfacial energies } \\
\text { 3. Diffusion coefficients } \\
\text { 4. Grain-boundary mobility }\end{array}$ \\
\hline Particle scale & $\begin{array}{l}\text { 1. Specific interfacial energies, diffusion } \\
\text { coefficients, grain-boundary mobility } \\
\text { 2. Initial microstructure } \\
\text { 3. temperature }\end{array}$ & 1. Microstructural evolution. \\
\hline $\begin{array}{l}\text { Component } \\
\text { scale }\end{array}$ & $\begin{array}{l}\text { 1. Constitutive and grain-growth laws, and their } \\
\text { parameters } \\
\text { 2. Initial density, average particle size and } \\
\text { component geometry } \\
\text { 3. Temperature and boundary conditions }\end{array}$ & $\begin{array}{l}\text { Temporal evolution of } \\
\text { 1.density and grain-size fields } \\
\text { 2.component deformation }\end{array}$ \\
\hline
\end{tabular}

Effort is being made to integrate these models into a multiscale sintering model. Fig. 1 shows our strategy for the multiscale modelling. The aim is to allow information shown in the right column of Fig. 1 to be taken into account. These variables are critical to the quality control of advanced ceramic products but are not currently considered in the continuum finite element analysis of sintering. Not all the integrations shown in Fig. 1 have been achieved. In the following sections, examples are provided to show our recent progress in the model integration.

A MULTI-SCALE SINTERING MODEL

EXTRA INPUT OF INTEGRATED MODEL

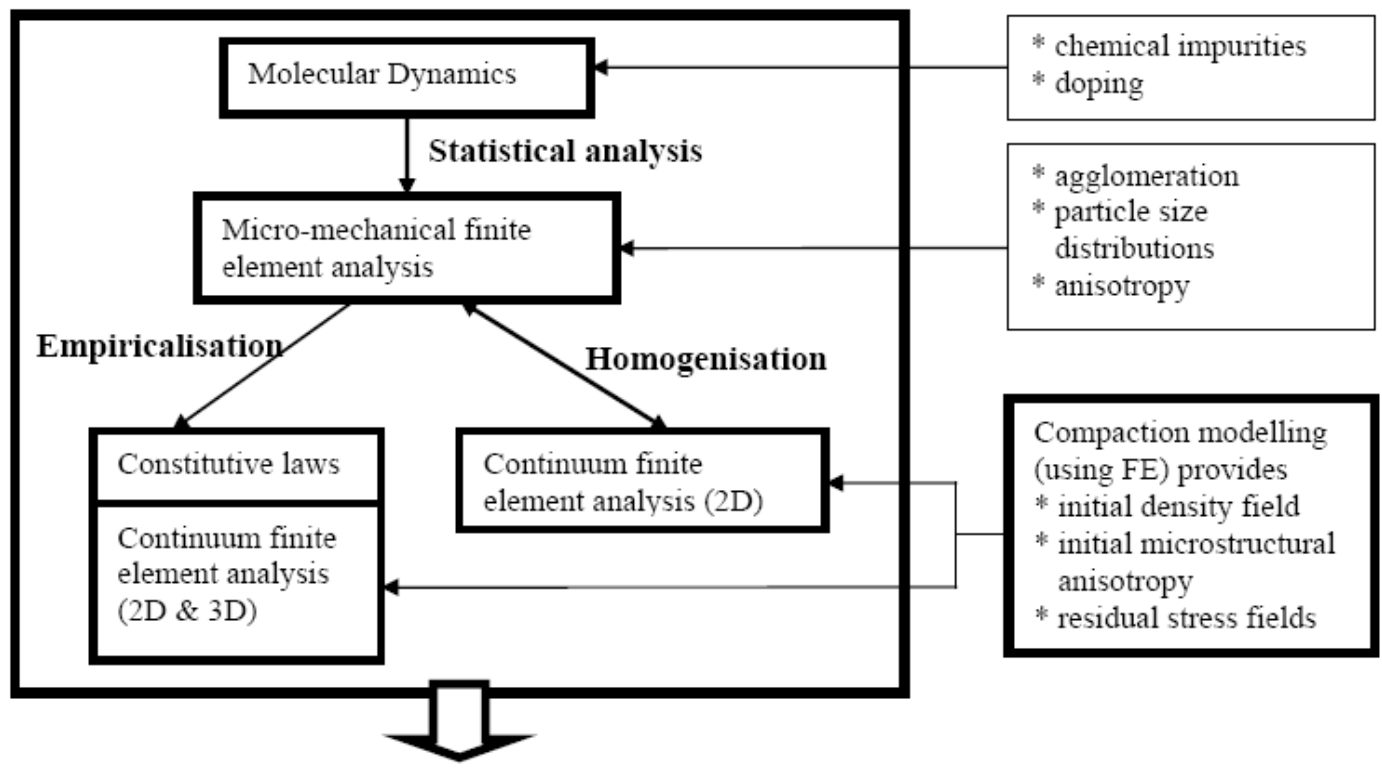

Sintering deformation; Density, grain size, and residual stress fields

Fig. 1. Flowchart for the integration of sintering models at atomistic, particle and continuum levels, and with powder compaction models.

\section{A local-global model for pore evolution}

It is possible to couple a particle scale model with a continuum scale model as shown in Fig. 2. Locally around the pore (inside the dashed circle) the detailed microstructural evolution is followed 
while in the remote region (marked by $B$ ) the material is assumed to be continuum and deform by viscous flow governed by Eqn. (1). The microstructural evolution is governed by the laws of solidstate diffusion and grain-boundary migration while the deformation in the continuum region is governed by the constitutive law. The two regions are connected through a variational principle initially used for diffusional creep crack propagation by Needleman and Rice [8].

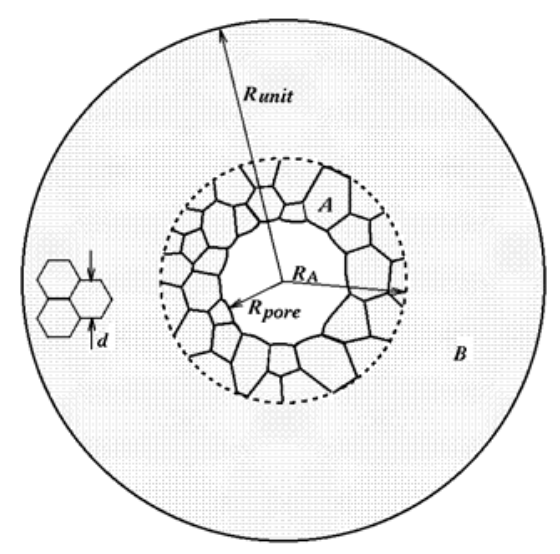

Fig. 2. A local-global model combining particle and continuum models

Fig. 3 shows a simple example of computer simulation using the local and global model. In this simple case, the continuum region is assumed to deform by Coble creep and the pore is assumed to be surrounded by identical grains. Only the local model is shown in the figure. The model predicts that the pore grows instead of shrinking in consistence with Kingery's theory.

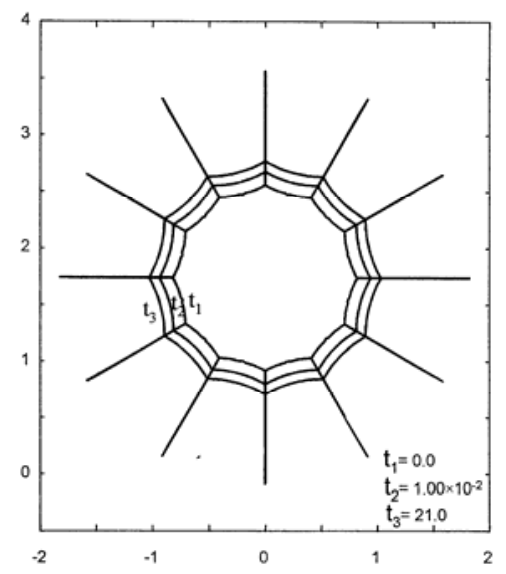

Fig. 3. Computer simulated evolution of a large pore using the local-global model.

The Kingery's theory is however invalid if the grains surrounding the large pore are non-uniform as in practice. Ref. [9] provides a full discussion for the sintering kinetics of large pores.

\section{On-line constitutive law}

Traditionally constitutive laws (Hooke's law for example) and the finite element method were developed separately. An analytical expression of the constitutive law is developed first and then implemented into a finite element computer programme. A unique feature of the sintering constitutive law is that the parameters in the law and even the law itself change significantly as the density and grain size increase during sintering. A so-called on-line constitutive law has been proposed to address this issue, first by Olevsky and et. al. [10] and later improved by Huang and Pan [11]. Instead of using a pre-established constitutive law, the constitutive response of the material is "derived" numerically and simultaneously as a finite element analysis is being carried out. To do so, a micromechanical (particle) model is adopted at each integration point of a continuum finite element model. At each 
timestep, the macroscopic state of the material is fed into the micromechanical model which returns a corresponding constitutive response (providing viscosities and sintering potential). Fig. 4 shows computer simulated density distribution in a sintered material containing hard inclusions. Symmetry was assumed so that only a quarter of the model is shown. In this problem, because of the constraints by the large and rigid particles (the dark and rectangular regions), the small powder particles may either separate or approach each other depending on the macroscopic strain state. Very different constitutive responses can be expected in the separating and the approaching cases. On the other hand the macroscopic strain state can only be determined if the constitutive law of the powder matrix is known. The constitutive response and the macroscopic deformation are therefore interconnected. Using the on-line approach, the density evolution of the matrix material can be predicted as shown in the figure. More details of the numerical scheme can be found in [11].

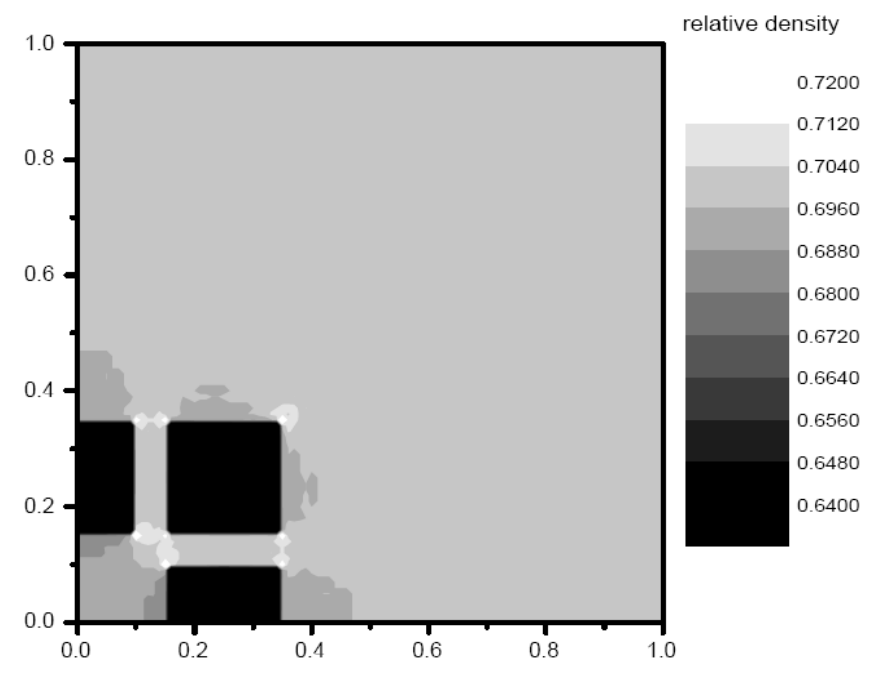

Fig. 4. Computer simulated density distribution in a powder material containing rigid inclusions. Only a quarter of the model is shown.

5. Finite element calculation of sintering deformation without a constitutive law

While the constitutive laws based on micromechanical models are being improved, it has been shown that a full constitutive law may not be necessary for a "proof of concept" finite element analysis [12]. It is possible to use only the densification data (density as a function of time), which is part of the constitutive response, in a finite element analysis to predict the sintering deformation to a good accuracy. Fig. 5 compares finite element predictions of sintering deformation obtained using the densification data (labelled as DFEM) and the full constitutive law of Eqn. (1) respectively.

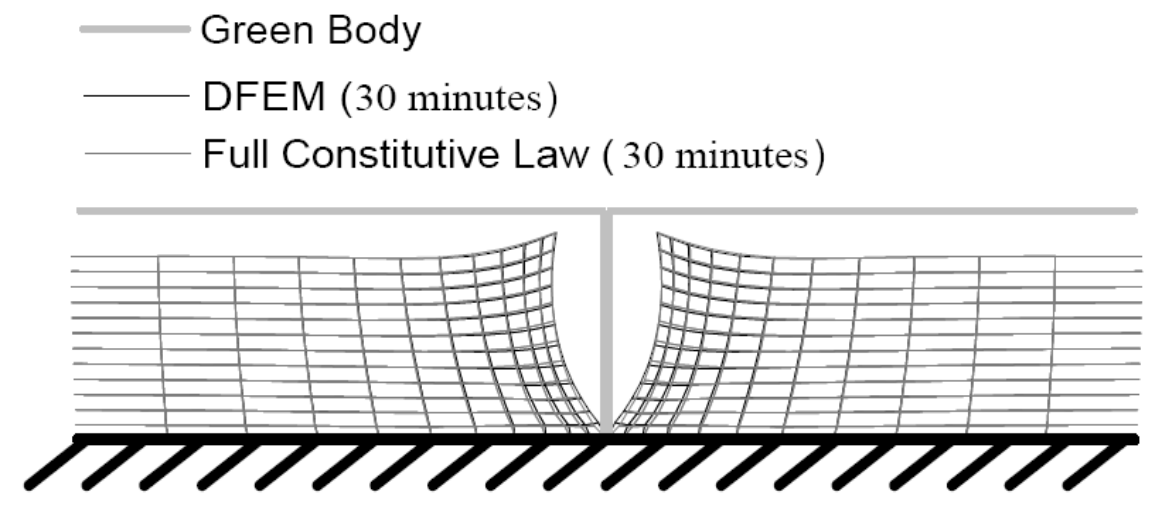

Fig. 5. Finite element predictions of sintering deformation for a cracked film on a rigid substrate obtained using the full constitutive law (Eqn. 1) and densification data respectively. 
An initially cracked thin film is constrained by a rigid substrate. The figure shows the predicted profile of the film after 30 minutes of sintering. It can be seen that a full constitutive law, which is difficult to calibrate as discussed in section 2, is unnecessary. Experimental case studies can be found in [12] which showed that finite element analysis using the densification data alone can give good predictions for both solid state and liquid phase sintering.

\section{Concluding remark}

Due to several generations of research, our fundamental understanding of the sintering phenomenon is clear. However our ability to predict the microstructure evolution and component deformation during the sintering process is still not yet matured enough for the computer models to be used routinely by the ceramic industry. The multiscale modelling approach may bring us one step closer to achieve this aim. In the meantime empirical approaches, like the one reported in ref. [12], can be used at least as a tool for a "proof of concept" material design.

Acknowledgement - This work is supported by an EPSRC research grant S57996 which is gratefully acknowledged.

\section{References}

1. Jingzhe Pan (2003), Modelling sintering at different length scales, International Materials Reviews, Vol. 48, pp. 69-85.

2. M.R. Zachariah and M.J. Carrier (1999), Molecular dynamics computation of gas-phase nanoparticle sintering: a comparison with phenomenological models, Journal of Aerosol Science, Vol. 30, pp. 1139-1151

3. C.L. Martin, L.C.R. Schneider, L. Olmos and D. Bouvard (2006), Discrete element modeling of metallic powder sintering, Scripta Materialia, Vol. 55, pp. 425-428.

4. H.N. Ch'ng and Jingzhe Pan (2004), Cubic Spline Elements for Modelling Microstructural Evolution of Materials Controlled by Solid-state Diffusion and Grain-boundary Migration, Journal of Computational Physics, Vol. 196, pp 724-750.

5. H.N. Ch'ng and Jingzhe Pan (2005), Modelling Microstructural Evolution of Porous Polycrystalline Materials and a Numerical Study of Anisotropic Sintering, Journal of Computational Physics, Vol. 204, Pages 430-461.

6. H. G. Kim, O. Gilla and D. Bouvard (2003), A Phenomenological Constitutive Model for Sintering of Alumina Powder, J. Euro. Ceram. Soc. 2003, 23, 1675-1685.

7. A.C.F. Cocks and I.C. Sinka (2007), Constitutive modelling of powder compaction - I. Theoretical concepts, Mechanics of Materials, Vol. 39, 2007, pp. 392-40.

8. A. Needleman and J.R. Rice (1980), Plastic creep flow effects in the diffusive cavitation of grain boundaries, Acta Metallurgica. Vol. 28, pp.1315-1332.

9. Jingzhe Pan, H.N. Ch'ng and A.C.F. Cocks (2005), Sintering Kinetics of Large Pores, Vol. 37, pp. 705-721, Mechanics of Materials.

10.E.A. Olevsky, A. Maximenko and O. Van Der Biest (2002), On-line sintering strength of ceramics composites, International Journal of Mechanical Science, vol. 44, 755-771.

11.R. Huang and J. Pan (2007), A Two-scale Model for Sintering Damage in Powder Compact Containing Inert Inclusions, Mechanics of Materials, vol. 39, pp710-726.

12.S. Kiani, J. Pan, J.A. Yeomans, M. Barriere and P. Blanchart (2007), Finite element analysis of sintering deformation using densification data instead of a constitutive law, vol. 27, pp2377-2383, Journal of the European Ceramic Society. 